\title{
Lenvatinib Combined with Anti-PD-I Antibodies Plus Transcatheter Arterial Chemoembolization for Unresectable Hepatocellular Carcinoma: A Multicenter Retrospective Study
}

Jia-Yi Wu, ${ }^{1,2, *}$ Zhen-Yu Yin, ${ }^{3, *}$ Yan-Nan Bai, ${ }^{1,2}$ Yu-Feng Chen, ${ }^{4}$ Song-Qiang Zhou, ${ }^{1,2}$ Shuang-Jia Wang, (1D $)^{5}$ Jian-Yin Zhou, (D) ${ }^{6}$ Yi-Nan Li, ${ }^{2}$ Fu-Nan Qiu, ${ }^{1,2}$ Bin $\mathrm{Li}^{5}$ Mao-Lin Yan ${ }^{1,2}$

'Shengli Clinical Medical College of Fujian Medical University, Fuzhou, Fujian Province, 35000I, People's Republic of China; ${ }^{2}$ Department of Hepatobiliary Pancreatic Surgery, Fujian Provincial Hospital, Fuzhou, Fujian Province, 35000I, People's Republic of China; ${ }^{3}$ Department of Hepatobiliary Surgery, Xiamen Traditional Chinese Medical Hospital, Xiamen, Fujian Province, 361000, People's Republic of China; ${ }^{4}$ Department of Hepatobiliary Surgery, The Zhangzhou Affiliated Hospital of Fujian Medical University, Zhangzhou, Fujian Province, 363000, People's Republic of China; ${ }^{5}$ Department of Hepato-Biliary-Pancreatic and Vascular Surgery, The First Affiliated Hospital of Xiamen University, Xiamen, Fujian Province, 361000, People's Republic of China; ${ }^{6}$ Department of Hepatobiliary Surgery, Zhongshan Hospital of Xiamen University, Xiamen, Fujian Province, 361000, People's Republic of China

*These authors contributed equally to this work

Correspondence: Mao-Lin Yan; Bin Li Tel +86 059l-882I III30; +86 13606056652

Fax +86 059I-87557768; +86 05922132222

Email yanmaolin74@।63.com; wasalee@I26.com
Background: Lenvatinib (LEN) combined with anti-PD-1 antibodies (PD-1) exerted promising effects on unresectable hepatocellular carcinoma (uHCC). We assessed the safety and clinical efficacy of triple therapy [LEN+PD-1+transcatheter arterial chemoembolization (TACE)] in uHCC.

Methods: uHCC patients with an ECOG PS score of $0-1$ and Child-Pugh class A who underwent triple therapy were included. The primary endpoint was objective response rate (ORR) based on mRECIST. Secondary endpoints were conversion rate to liver resection and treatment-related adverse events.

Results: Between November 2018 and December 2020, 62 uHCC patients who underwent triple therapy at four major cancer centers in China were analyzed, including 35 in BCLC-C, 21 in BCLC-B, and 6 in BCLC-A. With a median follow-up of 12.2 months (range, 7.6-33.3 months), the investigator and blinded independent central review-assessed ORR were $80.6 \%$ and $77.4 \%$, respectively. A total of 33 patients $(53.2 \%)$ reached the standard of conversion to resectable HCC and 29 patients underwent resection. The median interval between start of triple therapy and resection was 123 days (range, 55-372 days). Pathological complete response and major pathological response were observed in 16 and 24 patients, respectively. Median overall survival and progression-free survival were not reached. Treatment-related adverse events occurred in $74.2 \%$ of the patients (grade $\geq 3,14.5 \%$; grade $\geq 4,4.8 \%$ ).

Conclusion: Combination of LEN, PD-1 and TACE showed a high rate of tumor response and convert resection in uHCC patients, with manageable toxicity.

Keywords: unresectable hepatocellular carcinoma, tumor response, adverse events, combination therapy, monotherapy

\section{Introduction}

Hepatocellular carcinoma (HCC) is the second leading cause of cancer-related deaths in the world, with a 5-year survival rate below $10 \%$ for all stages. ${ }^{1,2}$ Surgical resection is the best choice for long-term survival, and a potential cure for HCC patients. ${ }^{3-6}$ However, due to the insidious onset, approximately $80 \%$ of HCC patients are in intermediate or advanced stages at diagnosis and are unsuitable candidates for curative surgery, leading to poor prognosis. ${ }^{7,8}$ Currently, transarterial chemoembolization (TACE) and systemic therapy are standard treatments for patients with intermediate and advanced stage $\mathrm{HCC}$, respectively. ${ }^{8-12}$ 
In the last 10 years, the rapid development of systemic therapy [tyrosine kinase inhibitors (TKIs) and immune checkpoint inhibitors (ICIs)] has shown dramatic therapeutic effects and brought new hope to the treatment of patients with unresectable HCC (uHCC). ${ }^{13}$ Moreover, combination therapy has shown better trend in tumor response and survival outcomes with monotherapy. ${ }^{13-18}$ Combined therapy with different mechanisms of action may improve outcomes. At present, most of the combination therapies are dual therapies: combinations of TKIs and ICIs, combinations of two ICIs, combinations of TKIs and TACE, combinations of ICIs and cytotoxic agent, or a combination of TKIs and hepatic arterial infusion chemotherapy. ${ }^{13-26}$ Notably, lenvatinib (LEN, TKIs) combined with anti-PD-1 antibodies (PD-1, ICIs) exerted promising effects on $\mathrm{uHCC}$, with an objective response rate (ORR) of 36-66.7\% (NCT03006926, NCT03418922, and NCT03841201). However, the clinical experience of triple therapy (TKIs + ICIs + TACE) in the treatment of patients with uHCC is limited. The purpose of this study was to assess the safety and clinical efficacy of triple therapy (LEN + PD-1 + TACE) in uHCC.

\section{Patients and Methods}

\section{Patients}

A retrospective study was conducted on uHCC patients who received triple therapy (LEN+PD-1+TACE) between November 2018 and December 2020 at four major cancer centers in China: the Fujian Provincial Hospital, the First Affiliated Hospital of Xiamen University, the Zhongshan Hospital of Xiamen University, and the Zhangzhou Affiliated Hospital of Fujian Medical University. The clinical and pathological data related to this study were prospectively entered into a database and retrospectively analyzed. In accordance with the Declaration of Helsinki, this study was approved by the Institutional Ethics Committee of each participating institution, and informed consent was obtained from all patients for their data to be used for research purposes.

The diagnosis of HCC was based on histological examination of tumor tissue obtained by percutaneous needle biopsy or clinico-radiological criteria according to guidelines proposed by the China Liver Cancer Staging ${ }^{27}$ as follows: 1) two radiological imaging assessments showing typical features of HCC (early enhancement in the arterial phase and early wash-out in the portal venous phase), 2) one radiological imaging assessment showing the typical features of $\mathrm{HCC}$ along with a serum alpha-fetoprotein (AFP) level $>400 \mathrm{ng} / \mathrm{mL}$. uHCC was defined as extensive bilobar involvement of the liver due to large solitary or multiple tumors, or invasion of major vessels including the main trunk of portal vein $(\mathrm{Vp} 4)$ and inferior vena cava (Vv3) according to the Liver Cancer Study Group of Japan. ${ }^{28}$ Conversion to resectable HCC was defined as follows: 1) R0 resection with preservation of a sufficient remnant liver volume is achievable; 2) Child-Pugh class A; 3) Eastern Cooperative Oncology Group performance status (ECOG-PS) score $0-1 ; 4$ ) no extrahepatic lesions; 5) no tumor thrombus in the main trunk of portal vein and inferior vena cava; and 6) no contraindications for hepatectomy.

The inclusion criteria were as follows: 1) uHCC patients treated with triple therapy; 2) aged between 18 and 75 years, with good operative tolerance; 3) patients must have at least one target lesion with measurable diameter and arterial enhancement according to modified Response Evaluation Criteria in Solid Tumors (mRECIST). ${ }^{29}$ The exclusion criteria were as follows: 1) Child-Pugh class B or C; 2) ECOG-PS score $>1$; 3) prior treatment with TACE or systemic therapy; 4) combined with other anticancer treatments, such as radiofrequency ablation, radiotherapy, and chemotherapy; 5) a history of other cancers; and 6) incomplete data.

Prior to treatment administration, all patients underwent complete medical history recording, physical examination, blood profiles [including complete blood count, liver, renal, adrenal, thyroid, cardiac, coagulation function tests, detection of hepatitis B surface antigen ( $\mathrm{HBsAg}$ ), HBV-DNA quantification, AFP and protein induced by vitamin $\mathrm{K}$ absence-II (PIVKA-II)], urine routine, and radiological examinations (abdominal contrast-enhanced CT and/or MRI).

\section{Procedures}

All patients received LEN ( $8 \mathrm{mg}$ for bodyweight $<60 \mathrm{~kg}$ or $12 \mathrm{mg}$ for bodyweight $\geq 60 \mathrm{~kg}$ ) orally once daily and PD- 1 (sintilimab $200 \mathrm{mg}$, tislelizumab $200 \mathrm{mg}$, camrelizumab $200 \mathrm{mg}$, toripalimab $240 \mathrm{mg}$, or pembrolizumab $200 \mathrm{mg}$ ) intravenously once every 3 weeks. Meanwhile, TACE was performed every 4-6 weeks if there was obvious hepatic arterial blood supply to HCC according to contrastenhanced abdominal CT or MRI. LEN and PD-1 were stopped for 3 days before and after TACE. All patients with active $\mathrm{HBV}$ infection received oral antiviral treatment (entecavir). 
Access for TACE was performed under local anesthesia via the right femoral artery. After the artery supplying the tumor was identified by selective hepatic angiography procedure, iodized oil and pirarubicin were mixed and injected into the selected tumor artery through the microcatheter. Subsequently, the feeding arteries were selectively embolized with gelatin sponge particles until complete arterial flow stasis was observed.

\section{Response and Toxicity Evaluation}

The tumor response was assessed as complete response (CR), partial response (PR), stable disease (SD), or progressive disease $(\mathrm{PD})$ via contrast-enhanced $\mathrm{CT}$ or MRI every $4-8$ weeks according to mRECIST criteria both by the investigator and blinded independent central review (BICR). The ORR was defined as the proportion of patients with the best response (either $\mathrm{CR}$ or $\mathrm{PR}$ ) $\geq 4$ weeks after the criteria for response were first met, while the disease control rate (DCR) was defined as CR, PR, and $\mathrm{SD}$. Time to response was calculated from the initiation of triple therapy until the date when the criteria for PR or $\mathrm{CR}$ were first met. Pathologic $\mathrm{CR}$ and major pathologic response were defined as the complete absence and less than or equal to $10 \%$ of viable tumor cells in the resected specimen, respectively. ${ }^{27}$

Toxicities were extracted for analysis from the hospital electronic medical records according to the Common Terminology Criteria for Adverse Events, Version 5.0.

\section{Follow-Up}

Patients were followed-up every 4-8 weeks. At each appointment, clinical, laboratory, and radiological (contrast-enhanced CT and/or MRI) data were collected. Once the patient reached the standard of conversion to resectable HCC, curative intent resection and postoperative adjuvant treatment were performed after informed consent. LEN was stopped for 1 week and PD-1 for 1 month before and after resection. Other patients were treated until PD, symptomatic progression, intolerable toxicity, or withdrawal of consent. The choice of the subsequent treatment was determined after discussions by a multi-disciplinary team and considering the patient's preference. Patients who underwent surgical resection received systemic therapy (LEN + PD-1) for 3-6 months.

The primary endpoint of this study was ORR assessed by the investigator and blinded independent central review (BICR) per mRECIST. The secondary endpoints were conversion rate to liver resection and treatment-related adverse events (TRAEs). The endpoint of follow-up was July 1, 2021.

\section{Statistical Analysis}

Continuous data were non-normal (on KolmogorovSmirnov test) and expressed as median (range). Categorical data were expressed as $\mathrm{n}$ (percentage). All statistical analyses were performed using Statistical Package for Social Sciences (SPSS) software (Version 23, SPSS, Inc., Chicago, IL, USA).

\section{Results \\ Patient Characteristics}

Between November 2018 and December 2020, a total of 62 patients received triple therapy (LEN+PD-1+TACE) at four major cancer centers in China were enrolled. Of 62 patients (median age, 57 years; range, 23-75 years; 56 men and 6 women), 6 patients with BCLC stage A, 21 patients with BCLC stage $\mathrm{B}$, and 35 patients with BCLC stage C; 57 patients $(91.9 \%)$ had hepatitis B virus, 32 patients $(51.6 \%)$ had baseline AFP $\geq 400 \mathrm{ng} / \mathrm{mL}, 45$ patients $(72.6 \%)$ had baseline PIVKA-II $\geq 400 \mathrm{mAU} / \mathrm{mL}$. The median size of the baseline target lesions was $9.7 \mathrm{~cm}$ (range, 2-21.7 cm). Macrovascular invasion was present in 34 patients $(54.8 \%)$, extrahepatic metastases in 6 patients (9.7\%), and both in 5 patients (8.1\%). Patient demographics and baseline characteristics are listed in Table 1.

The PD-1s used were sintilimab (200 mg, $\mathrm{n}=22$ ), tislelizumab (200 mg, $\mathrm{n}=15)$, camrelizumab (200 mg, n=11), toripalimab (240 $\mathrm{mg}, \mathrm{n}=10)$, and pembrolizumab (200 mg, $\mathrm{n}=4$ ).

\section{Tumor Response and Safety}

During a median follow-up of 12.2 months (range, 7.633.3 months), best responses were $20 \mathrm{CR}, 30 \mathrm{PR}, 8 \mathrm{SD}$, and $4 \mathrm{PD}$ per investigator and $17 \mathrm{CR}, 31 \mathrm{PR}, 9 \mathrm{SD}, 4 \mathrm{PD}$, and 1 not evaluable per BICR according to mRECIST criteria, respectively. The investigator and BICR-assessed ORR were $80.6 \%$ (50/62) and $77.4 \%$ (48/62), respectively (Table 2). Median time to response was 55 days (range, 23-297 days). Mean duration of treatment with LEN was 52 days (range, 23-294 days). Median number of PD-1 and TACE were two cycles (range, 1-12 cycles) and two times (range, 1-7 times), respectively. Waterfall analysis showed tumor size reduction in $91.9 \%$ (57/62) of the patients as per investigator assessment (Figure 1). 
Table I Patient Demographics and Baseline Characteristics

\begin{tabular}{|c|c|}
\hline Characteristics & Patients $(\mathrm{N}=62)$ \\
\hline Median age, years $(\text { range })^{\mathrm{a}}$ & $57(23-75)$ \\
\hline \multicolumn{2}{|l|}{ Age, years, n (\%) } \\
\hline$<65$ & $50(80.6)$ \\
\hline$\geq 65$ & $12(19.4)$ \\
\hline \multicolumn{2}{|l|}{ Sex, n (\%) } \\
\hline Female & $6(9.7)$ \\
\hline Male & $56(90.3)$ \\
\hline \multicolumn{2}{|l|}{ ECOG PS, n (\%) } \\
\hline 0 & $60(96.8)$ \\
\hline 1 & $2(3.2)$ \\
\hline \multicolumn{2}{|l|}{ Etiology, n (\%) } \\
\hline Hepatitis B infection & $57(91.9)$ \\
\hline Unknown & $5(8.1)$ \\
\hline \multicolumn{2}{|l|}{ HBV-DNA copy, n (\%) } \\
\hline$<1000 \mathrm{copy} / \mathrm{mL}$ & $28(45.2)$ \\
\hline$\geq 1000 \mathrm{copy} / \mathrm{mL}$ & $34(54.8)$ \\
\hline \multicolumn{2}{|l|}{ Pre-treatment AFP, n (\%) } \\
\hline$<400 \mathrm{ng} / \mathrm{mL}$ & $30(48.4)$ \\
\hline$\geq 400 \mathrm{ng} / \mathrm{mL}$ & $32(51.6)$ \\
\hline \multicolumn{2}{|l|}{ Pre-treatment PIVKA-II, mAU/mL, n (\%) } \\
\hline$<400 \mathrm{mAU} / \mathrm{mL}$ & $17(27.4)$ \\
\hline$\geq 400 \mathrm{mAU} / \mathrm{mL}$ & $45(72.6)$ \\
\hline \multicolumn{2}{|l|}{ Tumor number, n (\%) } \\
\hline Solitary & $25(40.3)$ \\
\hline Multiple & $37(59.7)$ \\
\hline Median tumor size, $\mathrm{cm}$ (range) $)^{\mathrm{a}}$ & $9.7(2-21.7)$ \\
\hline \multicolumn{2}{|l|}{ Tumor size, cm, n (\%) } \\
\hline$<10$ & $31(50)$ \\
\hline$\geq 10$ & $31(50)$ \\
\hline \multicolumn{2}{|l|}{ Tumor Location, n (\%) } \\
\hline Unilobar & $31(50)$ \\
\hline Bilobar & $26(41.9)$ \\
\hline Central & $5(8.1)$ \\
\hline \multicolumn{2}{|l|}{ Portal vein invasion, $\mathrm{n}(\%)$} \\
\hline $\mathrm{V}_{\mathrm{p} 0}$ & $35(56.5)$ \\
\hline$V_{p 2}$ & $4(6.5)$ \\
\hline$V_{p} 3$ & $12(19.4)$ \\
\hline$V_{p} 4$ & II (I7.7) \\
\hline \multicolumn{2}{|l|}{ Hepatic vein tumor thrombus, $n(\%)$} \\
\hline Vvo & $47(75.8)$ \\
\hline $\mathrm{Vvl}$ & $7(11.3)$ \\
\hline Vv2 & $3(4.8)$ \\
\hline Vv3 & $5(8.1)$ \\
\hline Macrovascular invasion, $\mathrm{n}(\%)$ & $34(54.8)$ \\
\hline
\end{tabular}

(Continued)
Table I (Continued).

\begin{tabular}{|l|c|}
\hline Characteristics & Patients (N = 62) \\
\hline Extrahepatic metastasis, n (\%) & $6(9.7)$ \\
\hline BCLC staging, n (\%) & \\
A & $6(9.7)$ \\
B & $21(33.9)$ \\
C & $35(56.5)$ \\
\hline
\end{tabular}

Note: ${ }^{2}$ Continuous data with a normal distribution are expressed as mean \pm SD. Abbreviations: ECOG PS, Eastern Cooperative Oncology Group performance status; AFP, $\alpha$-fetoprotein; PIVKA-II, protein induced by vitamin $\mathrm{K}$ absence-II; BCLC, Barcelona Clinic Liver Cancer.

Table 2 Tumor Responses per Investigator and BICR Assessment (mRECIST)

\begin{tabular}{|l|c|c|}
\hline \multirow{2}{*}{ Best Response, $\mathbf{n}(\%)$} & \multicolumn{2}{|c|}{ Triple Therapy (n=62) } \\
\cline { 2 - 3 } & Investigator & BICR \\
\hline Complete response & $20(30.6)$ & $17(27.4)$ \\
Partial response & $30(50)$ & $31(50)$ \\
Stable disease & $8(12.9)$ & $9(14.5)$ \\
Progressive disease & $4(6.5)$ & $4(6.5)$ \\
Not evaluable & 0 & $1(1.6)$ \\
Objective response rate, $\mathrm{n}(\%)$ & $50(80.6)$ & $48(77.4)$ \\
Disease control rate, $\mathrm{n}(\%)$ & $58(93.5)$ & $57(91.9)$ \\
\hline
\end{tabular}

Abbreviations: BICR, blinded independent central review; mRECIST, modified Response Evaluation Criteria in Solid Tumors.

In six uHCC patients with BCLC stage A, three (50\%) and one $(16.7 \%)$ patient achieved CR and PR to yield an ORR of $66.7 \%$ per BICR. In 21 uHCC patients with BCLC stage B, 7 (33.3\%) and 9 (42.9\%) patients achieved $\mathrm{CR}$ and $\mathrm{PR}$ to give an ORR of $76.2 \%$ per BICR. In 35 uHCC patients with BCLC stage C, $7(20 \%)$ and $21(60 \%)$ patients achieved CR and PR to yield an ORR of $80 \%$ per BICR (Table 3).

TRAEs occurred in $46(74.2 \%)$ patients (grade $\geq 3$, $14.5 \%$; grade $\geq 4,4.8 \%$ ). Among them, the most frequent ( $\geq 10 \%$ of patients) were increased alanine aminotransferase, decreased appetite, increased aspartate aminotransferase, increased blood bilirubin, fatigue, hypertension, abdominal pain, diarrhea, proteinuria, and hand-foot skin reaction (Table 4). Three patients had grade 4 TRAEs [autoimmune hepatitis $(n=2)$ and duodenum perforation $(n=1)]$ and no patient had a grade 5 TRAE. All TRAEs were evaluated as mild and manageable during the follow-up.

\section{Convert Resection After Triple Therapy}

A total of 33 patients $(33 / 62,53.2 \%)$ reached the standard of conversion to resectable $\mathrm{HCC}$ ( 3 with BCLC A, 11 with 


\section{Best reduction from baseline in size of target lesions by investigator}

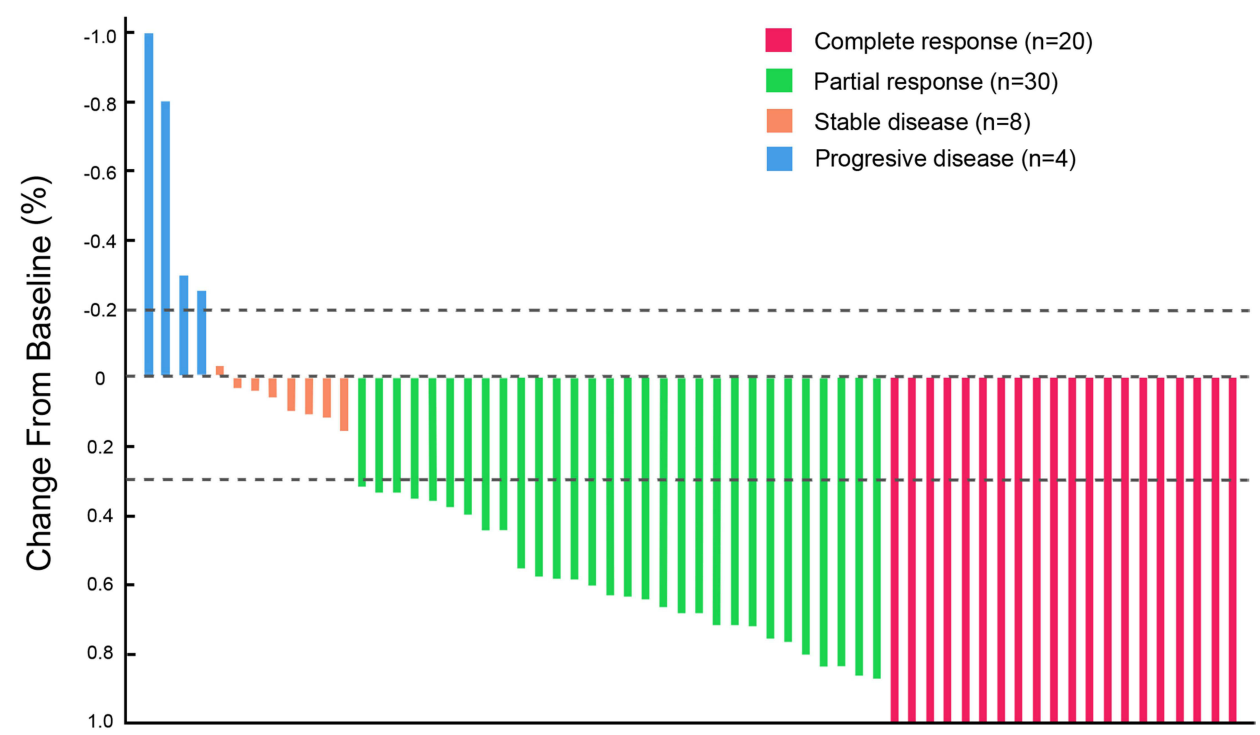

Figure I Waterfall plot of maximum tumor response to triple therapy by investigator using the mRECIST.

BCLC B, and 19 with BCLC C). Of them, four patients refused surgical resection and continued triple therapy, and the other 29 patients $(29 / 62,46.8 \%)$ underwent resection. The median interval between the start of triple therapy and resection was 123 days (range, 55-372 days). Pathological $\mathrm{CR}$ and major pathological response were observed in 16 $(16 / 62,25.8 \%)$ and 24 patients $(24 / 62,38.7 \%)$, respectively.

The median operation time and estimated blood loss volume were $235 \mathrm{~min}$ (range, 90-360 $\mathrm{min}$ ) and $300 \mathrm{~mL}$ (range, 100-6000 mL), respectively. Clavien-Dindo IIIb$\mathrm{V}$ complications occurred in three patients, including pulmonary embolism $(\mathrm{n}=1)$ and postoperative hepatic function failure $(n=2)$. One patient died of liver failure 9 days after surgery. The median postoperative hospital stay was 10 days (range, 6-61 days).

During a median follow-up of 250 days after surgery (range, 9-846 days), two patients developed intrahepatic recurrence (62 days and 171 days, respectively), one patient developed extrahepatic recurrence 334 days after surgery, and one patient died of liver failure 94 days after surgery. Mean duration of treatment with LEN after surgery was 152 days (range, $0-183$ days) and median number of PD-1 after surgery was six cycles (range, 0-7 cycles). Median overall survival (OS) and progressionfree survival (PFS) were not reached.

Table 3 Tumor Responses per Investigator and BICR Assessment (mRECIST)

\begin{tabular}{|c|c|c|c|c|c|c|}
\hline \multirow[t]{2}{*}{ Best Response, n (\%) } & \multicolumn{3}{|c|}{ Investigator $(n=62)$} & \multicolumn{3}{|c|}{ BICR $(n=62)$} \\
\hline & $\begin{array}{c}\text { BCLC-A } \\
(n=6)\end{array}$ & $\begin{array}{c}\text { BCLC-B } \\
(n=2 I)\end{array}$ & $\begin{array}{c}\text { BCLC-C } \\
(n=35)\end{array}$ & $\begin{array}{c}\text { BCLC-A } \\
(n=6)\end{array}$ & $\begin{array}{c}\text { BCLC-B } \\
(n=2 I)\end{array}$ & $\begin{array}{c}\text { BCLC-C } \\
(n=35)\end{array}$ \\
\hline Complete response & $3(50)$ & $9(42.9)$ & $8(22.9)$ & $3(50)$ & $7(33.3)$ & $7(20)$ \\
\hline Partial response & I (I6.7) & $9(42.9)$ & $20(57.1 \%)$ & I (I6.7) & $9(42.9)$ & $21(60)$ \\
\hline Stable disease & $2(33.3)$ & $2(9.5)$ & $4(I I .4)$ & $2(33.3)$ & $3(14.3)$ & $4(I I .4)$ \\
\hline Progressive disease & 0 & I (4.8\%) & $3(8.6)$ & 0 & I (4.8) & $3(8.6)$ \\
\hline Not evaluable & 0 & 0 & 0 & 0 & I (4.8) & 0 \\
\hline Objective response rate, $\mathrm{n}(\%)$ & $4(66.7)$ & $18(85.7)$ & $28(80)$ & $4(66.7)$ & $16(76.2)$ & $28(80)$ \\
\hline Disease control rate, n (\%) & $6(100)$ & $20(95.2)$ & $32(91.4)$ & $6(100)$ & $19(90.5)$ & $32(9 \mid .4)$ \\
\hline Conversion to resectable HCC, $\mathrm{n}(\%)$ & $3(50)$ & II (52.4) & $19(54.3)$ & $3(50)$ & II (52.4) & $19(54.3)$ \\
\hline
\end{tabular}

Abbreviations: BICR, blinded independent central review; mRECIST, modified Response Evaluation Criteria in Solid Tumors; BCLC, Barcelona Clinic Liver Cancer; HCC, hepatocellular carcinoma. 
Table 4 Common Adverse Events Occurring in $\geq 10 \%$ of Patients

\begin{tabular}{|c|c|c|c|}
\hline Adverse Event & Any Grade $(n=46)$ & Grade I-2 $(n=37)$ & Grade $3(n=6)$ \\
\hline Increased alanine aminotransferase & $38(61.3 \%)$ & $37(59.7 \%)$ & $\mathrm{I}(\mathrm{I} .6 \%)$ \\
\hline Decreased appetite & $35(56.5 \%)$ & $35(56.5 \%)$ & 0 \\
\hline Increased aspartate aminotransferase & $34(54.8 \%)$ & $33(53.2 \%)$ & I (I.6\%) \\
\hline Increased blood bilirubin & $22(35.5 \%)$ & $21(33.9 \%)$ & $\mathrm{I}(\mathrm{I} .6 \%)$ \\
\hline Fatigue & $18(29 \%)$ & $18(29 \%)$ & 0 \\
\hline Hypertension & $15(24.2 \%)$ & $14(22.6 \%)$ & $\mathrm{I}(\mathrm{I} .6 \%)$ \\
\hline Abdominal pain & $12(19.4 \%)$ & $12(19.4 \%)$ & 0 \\
\hline Diarrhea & $8(12.9 \%)$ & 7 (II.3\%) & $\mathrm{I}(\mathrm{I} .6 \%)$ \\
\hline Proteinuria & $8(12.9 \%)$ & 7 (II.3\%) & I (I.6\%) \\
\hline Hand-foot skin reaction & 7 (II.3\%) & $3(4.8 \%)$ & $4(6.5 \%)$ \\
\hline
\end{tabular}

\section{Discussion}

In this study, triple therapy showed a high ORR $(80.6 \%$ as per investigator and $77.4 \%$ as per BICR, respectively) and conversion rate to liver resection (46.8\%) with a manageable toxicity. Waterfall analysis showed tumor size reduction in $91.9 \%$ of the patients. Notably, although 4 patients refused surgical resection, there were 16 patients (16/62, 25.8\%) confirmed with pathological CR and 24 patients $(24 / 62,38.7 \%)$ confirmed with major pathological response. Meanwhile, the onset time of triple therapy was short, with a median TTR of 55 days. Almost half of the patients showed tumor response at the first review. During a median follow-up of 12.2 months (range, 7.6-33.3 months), median OS and PFS were not reached.

uHCC leads to great trauma in patients and a heavy psychological burden accompanies poor prognosis. Thus, fast-acting and effective treatments are urgently needed. At present, options for first-line therapy of advanced HCC include sorafenib, LEN, and atezolizumab plus bevacizumab; options for second-line therapy of advanced HCC include regorafenib, cabozantinib, pembrolizumab, nivolumab, and ramucirumab. In the SHARP trial, sorafenib was shown to prolong OS by approximately 3 months compared with placebo in patients with uHCC. However, the ORR of the sorafenib group (2\%) was relatively low. ${ }^{30}$ Similarly, monotherapy of ICIs in advanced HCC also resulted in limited ORR, ranging from $14 \%$ to $20 \% .^{13,31,32}$ In 2018, Phase III REFLECT trial demonstrated that LEN was non-inferior to sorafenib in terms of OS (13.6 months vs 12.3 months) with an ORR of $24.1 \%{ }^{33}$ To date, LEN has achieved relatively high ORR in monotherapy.

Numerous clinical trials in patients with uHCC have shown that combination of TKIs and ICIs achieved substantial clinical efficacy, with higher ORR and better survival outcomes than monotherapy. ${ }^{13-26}$ In 2020, the FDA approved the combination therapy of atezolizumab plus bevacizumab as a first-line treatment for advanced HCC based on the results of the IMbrave150 trial, which demonstrated an ORR of $33.2 \%$ (mRECIST) and median PFS of 6.8 months. ${ }^{19}$ The combination therapy of LEN and pembrolizumab showed good anti-tumor activity, with an impressive ORR of $46 \%$, median PFS of 9.3 months, and median OS of 22 months (NCT03006926). ${ }^{20}$ The ORR of dual therapy in uHCC ranged from $13.6 \%$ to $46 \% .^{13-26}$

TACE is the only guideline-recommended therapy for intermediate $\mathrm{HCC}$ patients and showed a high tumor response to uHCC with a tolerable safety profile. Combined therapy with different mechanisms of action may improve outcomes, ${ }^{34}$ thus we assessed the triple therapy (LEN+PD-1+TACE) in uHCC, which achieved the highest ORR, conversion rate to liver resection, and pathological CR rate to date. Notably, the patients in the present study were relatively more late-staged, and included cases with bilobar tumor location (41.9\%), macrovascular invasion $(54.8 \%)$, extrahepatic metastases $(9.7 \%)$, and tumor burden $>10 \mathrm{~cm}(50 \%)$. A total of 33 patients $(53.2 \%)$ reached the standard of conversion to resectable HCC and 29 patients $(46.8 \%)$ achieved surgical resection. Due to limited sample size and relatively short follow-up time, long-term outcomes and adverse effects of triple therapy need further study. Importantly, triple therapy was very effective in uHCC, with manageable toxicity.

The effect of triple therapy might be attributed to the following reasons. PD-1 blocks the signals that impede immune attacks on tumors to facilitate an effective immune response against tumor cells. ${ }^{31,32}$ TACE reduces 
the blood supply of HCC and activates the release of tumor-specific antigens, which will enhance the clinical efficacy of PD-1 antibodies. ${ }^{34,35}$ However, the hypoxic microenvironment resulting from TACE subsequently promotes the secretion of HIF-1 alpha, bFGF and VEGF, which leads to tumor angiogenesis, recurrence and metastasis. ${ }^{34,35}$ LEN is a multi-kinase inhibitor targeting vascular endothelial growth factor 1-3, fibroblast growth factor receptors 1-4, PDGFR a, RET, and KIT, which inhibits the proneoangiogenic and immunosuppressive effects of tumor microenvironments, as well as improves the clinical benefit of TACE and PD-1 antibodies. ${ }^{34,36}$ A hepa1-6 HCC model showed that LEN had immunomodulatory activity, and its combination with PD-1 antibodies could increase the populations of CD8 + T cells by decreasing the proportions of monocytes and macrophages as well as activating the interferon pathway, thereby showing superior antitumor activity compared with the single treatment. ${ }^{37}$ In summary, the synergistic effects of triple therapy result in better tumor response and survival outcomes. However, the mechanism of triple therapy is complex and needs further studies for better understanding.

The present study had several limitations. First, this was a retrospective study with a limited sample size and relatively short follow-up time, resulting in inevitable selection bias and relatively limited evidence, and the real incidence rates and severity of TRAEs were probably underestimated. Second, this study is a single-arm study with no control group, it was not possible to compare the effectiveness and safety of triple therapy with other therapy (such as TACE, LEN, PD-1, and systemic therapy). Therefore, well-designed randomized controlled trials are needed to further verify the benefits of triple therapy in uHCC patients. Third, various PD-1 inhibitors were used in this study, which influenced the uniformity of the treatment procedure. Fourth, this study was conducted at four major cancer centers in China with a high proportion of HBV-related uHCC cases, but triple therapy might not be generalizable to $\mathrm{uHCC}$ patients due to other etiologies.

Based on the results of this study, combination of LEN, PD-1 and TACE showed a high rate of tumor response and convert resection in $\mathrm{UHCC}$ patients with a manageable toxicity. Thus, triple therapy may be a potential new treatment option for uHCC patients.

\section{Funding}

This study was funded by the Natural Science Foundation of Fujian Province (2020J011105).

\section{Disclosure}

The authors report no conflicts of interest.

\section{References}

1. Bray F, Ferlay J, Soerjomataram I, et al. Global cancer statistics 2018: GLOBOCAN estimates of incidence and mortality worldwide for 36 cancers in 185 countries. CA Cancer J Clin. 2018;68 (6):394-424. doi:10.3322/caac. 21492

2. Fong ZV, Tanabe KK. The clinical management of hepatocellular carcinoma in the United States, Europe, and Asia: a comprehensive and evidence-based comparison and review. Cancer. 2014;120 (18):2824-2838. doi:10.1002/cncr.28730

3. Johnson PJ. Non-surgical treatment of hepatocellular carcinoma. HPB (Oxford). 2005;7(1):50-55. doi:10.1080/13651820410024076

4. Cherqui Q, Laurent A, Mocellin N, et al. Liver resection for transplantable hepatocellular carcinoma: long-term survival and role of secondary liver transplantation. Ann Surg. 2009;250(5):738-746. doi:10.1097/SLA.0b013e3181bd582b

5. Roayaie S, Jibara G, Tabrizian P, et al. The role of hepatic resection in the treatment of hepatocellular cancer. Hepatology. 2015;62 (2):440-451. doi:10.1002/hep. 27745

6. Park JW, Chen M, Colombo M, et al. Global patterns of hepatocellular carcinoma management from diagnosis to death: the BRIDGE study. Liver Int. 2015;35(9):2155-2166. doi:10.1111/ liv. 12818

7. Colecchia A, Schiumerini R, Cucchetti A, et al. Prognostic factors for hepatocellular carcinoma recurrence. World J Gastroenterol. 2014;20 (20):5935-5950. doi:10.3748/wjg.v20.i20.5935

8. Villanueva A, Longo DL. Hepatocellular carcinoma. $N$ Engl J Med. 2019;380(15):1450-1462. doi:10.1056/NEJMra1713263

9. European Association for the Study of the Liver. EASL Clinical Practice Guidelines: management of hepatocellular carcinoma. J Hepatol. 2018;69(1):182-236. doi:10.1016/j.jhep.2018.03.019.

10. Heimbach JK, Kulik LM, Finn RS, et al. AASLD guidelines for the treatment of hepatocellular carcinoma. Hepatology. 2018;67(1):3 58-380. doi:10.1002/hep.29086

11. Marrero JA, Kulik LM, Sirlin CB, et al. Diagnosis, staging, and management of hepatocellular carcinoma: 2018 practice guidance by the American Association for the Study of Liver Diseases. Hepatology. 2018;68:723-750. doi:10.1002/hep.29913

12. Omata M, Cheng AL, Kokudo N, et al. Asia-Pacific clinical practice guidelines on the management of hepatocellular carcinoma: a 2017 update. Hepatol Int. 2017;11(4):317-370. doi:10.1007/s12072-0179799-9

13. Luo XY, Wu KM, He XX. Advances in drug development for hepatocellular carcinoma: clinical trials and potential therapeutic targets. J Exp Clin Cancer Res. 2021;40(1):172. doi:10.1186/ s13046-021-01968-w

14. Llovet JM, Montal R, Sia D, et al. Molecular therapies and precision medicine for hepatocellular carcinoma. Nat Rev Clin Oncol. 2018;15 (10):599-616. doi:10.1038/s41571-018-0073-4

15. Bangaru S, Marrero JA, Singal AG. Review article: new therapeutic interventions for advanced hepatocellular carcinoma. Aliment Pharmacol Ther. 2020;51(1):78-89. doi:10.1111/apt.15573

16. Kudo M. Systemic therapy for hepatocellular carcinoma: latest advances. Cancers (Basel). 2018;10(11):412. doi:10.3390/cancers10 110412

17. Liu X, Qin S. Immune checkpoint inhibitors in hepatocellular carcinoma: opportunities and challenges. Oncologist. 2019;24(Supp11): S3-S10. doi:10.1634/theoncologist.2019-IO-S1-s01

18. Liu Z, Lin Y, Zhang J, et al. Molecular targeted and immune checkpoint therapy for advanced hepatocellular carcinoma. $J$ Exp Clin Cancer Res. 2019;38(1):447. doi:10.1186/s13046-019-1412-8 
19. Finn RS, Qin S, Ikeda M, et al. Atezolizumab plus bevacizumab in unresectable hepatocellular carcinoma. $N$ Engl J Med. 2020;382 (20):1894-1905. doi:10.1056/NEJMoa1915745

20. Finn RS, Ikeda M, Zhu AX, et al. Phase Ib study of lenvatinib plus pembrolizumab in patients with unresectable hepatocellular carcinoma. J Clin Oncol. 2020;38(26):2960-2970. doi:10.1200/JCO.20.00808

21. Xu J, Shen J, Gu S, et al. Camrelizumab in combination with apatinib in patients with advanced hepatocellular carcinoma (RESCUE): a nonrandomized, open-label, phase II trial. Clin Cancer Res. 2021;27(4):1003-1011. doi:10.1158/1078-0432.CCR-20-2571

22. Yau T, Kang YK, Kim TY, et al. Efficacy and safety of nivolumab plus ipilimumab in patients with advanced hepatocellular carcinoma previously treated with sorafenib: the checkmate 040 randomized clinical trial. JAMA Oncol. 2020;6(11):e204564. doi:10.1001/ jamaoncol.2020.4564

23. He M, Li Q, Zou R, et al. Sorafenib plus hepatic arterial infusion of oxaliplatin, fluorouracil, and leucovorin vs sorafenib alone for hepatocellular carcinoma with portal vein invasion: a randomized clinical trial. JAMA Oncol. 2019;5(7):953-960. doi:10.1001/jamaoncol.2019.0250

24. Mei J, Li SH, Li QJ, et al. Anti-PD-1 immunotherapy improves the efficacy of hepatic artery infusion chemotherapy in advanced hepatocellular carcinoma. J Hepatocell Carcinoma. 2021;8:167-176. doi:10.2147/JHC.S298538

25. Xie D, Sun Q, Wang X, et al. Immune checkpoint inhibitor plus tyrosine kinase inhibitor for unresectable hepatocellular carcinoma in the real world. Ann Transl Med. 2021;9(8):652. doi:10.21037/atm-20-7037

26. Meyer T, Fox R, Ma YT, et al. Sorafenib in combination with transarterial chemoembolisation in patients with unresectable hepatocellular carcinoma (TACE 2): a randomised placebo-controlled, double-blind, phase 3 trial. Lancet Gastroenterol Hepatol. 2017;2 (8):565-575. doi:10.1016/S2468-1253(17)30156-5

27. Zhou J, Sun H, Wang Z, et al. Guidelines for the diagnosis and treatment of hepatocellular carcinoma (2019 Edition). Liver Cancer. 2020;9(6):682-720. doi:10.1159/000509424

28. Kudo M, Izumi N, Kokudo N, et al. Management of hepatocellular carcinoma in Japan: consensus-based clinical practice guidelines proposed by the Japan Society of Hepatology (JSH) 2010 updated version. Dig Dis. 2011;29(3):339-364. doi:10.1159/000327577
29. Lencioni R, Llovet JM. Modified RECIST (mRECIST) assessment for hepatocellular carcinoma. Semin Liver Dis. 2010;30(1):52-60. doi:10.1055/s-0030-1247132

30. Llovet JM, Ricci S, Mazzaferro V, et al. Sorafenib in advanced hepatocellular carcinoma. $N$ Engl J Med. 2008;359(4):378-390. doi:10.1056/NEJMoa0708857

31. Elkhoueiry AB, Sangro B, Yau T, et al. Nivolumab in patients with advanced hepatocellular carcinoma (CheckMate 040): an open-label, non-comparative, phase $1 / 2$ dose escalation and expansion trial. Lancet. 2017;389(10088):2492-2502. doi:10.1016/S0140-6736(17) 31046-2

32. Zhu AX, Finn RS, Edeline J, et al. Pembrolizumab in patients with advanced hepatocellular carcinoma previously treated with sorafenib (KEYNOTE-224): a non-randomised, open-label phase 2 trial. Lancet Oncol. 2018;19(7):940-952. doi:10.1016/S1470-2045(18) 30351-6

33. Kudo M, Finn RS, Qin S, et al. Lenvatinib versus sorafenib in first-line treatment of patients with unresectable hepatocellular carcinoma: a randomised phase 3 non-inferiority trial. Lancet. 2018;391 (10126):1163-1173. doi:10.1016/S0140-6736(18)30207-1

34. Hack SP, Zhu AX, Wang Y. Augmenting anticancer immunity through combined targeting of angiogenic and PD-1/PD-L1 pathways: challenges and opportunities. Front Immunol. 2020;11: 598877. doi:10.3389/fimmu.2020.598877

35. Kudo M. A new treatment option for intermediate-stage hepatocellular carcinoma with high tumor burden: initial lenvatinib therapy with subsequent selective TACE. Liver Cancer. 2019;8(5):299-311. doi:10.1159/000502905

36. Yamamoto Y, Matsui J, Matsushima T, et al. Lenvatinib, an angiogenesis inhibitor targeting VEGFR/FGFR, shows broad antitumor activity in human tumor xenograft models associated with microvessel density and pericyte coverage. Vasc Cell. 2014;6:18. doi:10.1186/ 2045-824X-6-18

37. Kimura T, Kato Y, Ozawa Y, et al. Immunomodulatory activity of lenvatinib contributes to antitumor activity in the Hepa1-6 hepatocellular carcinoma model. Cancer Sci. 2018;109(12):399-4002. doi:10. 1111/cas. 13806
Journal of Hepatocellular Carcinoma

\section{Publish your work in this journal}

The Journal of Hepatocellular Carcinoma is an international, peerreviewed, open access journal that offers a platform for the dissemination and study of clinical, translational and basic research findings in this rapidly developing field. Development in areas including, but not limited to, epidemiology, vaccination, hepatitis therapy, pathology and molecular tumor classification and prognostication are all considered for publication. The manuscript management system is completely online and includes a very quick and fair peer-review system, which is all easy to use. Visit http://www.dovepress.com/ testimonials.php to read real quotes from published authors. 